\title{
Correlation transfer equation for multiply scattered light modulated by an ultrasonic pulse: an analytical model and Monte Carlo simulation
}

Sava Sakadži?, Lihong V. Wang

Sava Sakadži?, Lihong V. Wang, "Correlation transfer equation for multiply scattered light modulated by an ultrasonic pulse: an analytical model and Monte Carlo simulation," Proc. SPIE 6437, Photons Plus Ultrasound: Imaging and Sensing 2007: The Eighth Conference on Biomedical Thermoacoustics, Optoacoustics, and Acousto-optics, 64371K (13 February 2007); doi: $10.1117 / 12.702600$ 


\title{
Correlation transfer equation for multiply scattered light modulated by an ultrasonic pulse: An analytical model and Monte Carlo simulation
}

\author{
Sava Sakadžić and Lihong V. Wang \\ Optical Imaging Laboratory, Department of Biomedical Engineering, \\ Texas A\&M University, College Station, Texas 77843-3120
}

\begin{abstract}
We present derivation of the temporal correlation transfer equation (CTE) for multiply scattered light modulated by an ultrasound pulse. The equation can be used to obtain the time-varying specific intensity of light produced by a pulsed and nonuniform ultrasound field in optically scattering media that have a heterogeneous distribution of optical parameters. We also develop a Monte Carlo algorithm that can simulate the spatial distribution of the time-dependent power spectrum density of light modulated by a focused ultrasound pulse in optically scattering media with heterogeneous distributions of optically scattering and absorbing objects. Derivation is based on the ladder diagram approximation of the Bethe-Salpeter equation that assumes moderate ultrasound pressures. We expect these results to be applicable to a wide spectrum of conditions in the ultrasound-modulated optical tomography of soft biological tissues.
\end{abstract}

Keywords: ultrasound-modulated optical tomography, multiply scattered light, correlation transfer equation, Bethe-Salpeter equation, Dyson equation

\section{INTRODUCTION}

Ultrasound-modulated optical tomography (UOT) is a hybrid technique that combines the advantages of ultrasonic resolution and optical contrast. ${ }^{1,2}$ In this technique, optical radiation and focused ultrasound are simultaneously applied to soft biological tissue and the resulting ultrasound-modulated light is measured. The measurement provides information about the optical properties of the tissue, spatially localized at the interaction region of the ultrasonic and the electromagnetic waves. Images can be created by scanning either the ultrasound or the tissue sample..$^{3,4}$

A theoretical understanding of the ultrasound modulation of multiply scattered light is still incomplete. Present theoretical models include two mechanisms of modulation. The first mechanism accounts for dynamic scattering by optical scatterers oscillating in an ultrasound field, which causes optical frequency shifts. ${ }^{5,6}$ The second mechanism accounts for ultrasound-induced changes in the optical index of refraction..$^{7,8}$ Both mechanisms have been combined by Wang, ${ }^{8}$ in a model based on the diffusing-wave spectroscopy (DWS) approach..$^{9,10}$ Subsequently, the equations are extended to account for anisotropic optical scattering ${ }^{11}$ and Brownian motion. ${ }^{11,12}$ In addition, Ref. 13 accounts for pulsed ultrasound and strong correlations between ultrasound-induced optical phase increments, which exist when the ratio of the optical transport mean-free path $l_{t r}$ to the ultrasound wavelength $\lambda_{a}$ is small. In the case of anisotropic optical scattering, equations derived for the isotropic case can be applied by substituting $l_{t r}$ for the optical mean-free path $l_{s} .{ }^{11,13}$ However, the applications of theoretical models based on the DWS approach are limited to simple geometries where it is possible to approximate the ultrasound field within the sample with a plane ultrasound wave and where the probability density function of the optical path length between the source and detector is analytically known. In most experiments a focused ultrasound beam is used and the sample geometries are complex with heterogeneously distributed optical parameters.

A more general theoretical model based on the ladder diagram approximation of the Bethe-Salpeter equation was developed recently. ${ }^{14,15}$ A temporal correlation transfer equation $(\mathrm{CTE})^{16-19}$ was derived for multiply scattered light modulated by continuous-wave $(\mathrm{CW})$ ultrasound. This equation can be used to obtain the timevarying specific intensity and subsequently the power spectral density of light produced by a focused ultrasound

Photons Plus Ultrasound: Imaging and Sensing 2007: The Eighth Conference on Biomedical Thermoacoustics, Optoacoustics, and Acousto-optics, edited by Alexander A. Oraevsky, Lihong V. Wang, Proc. of SPIE Vol. 6437, 64371K, (2007) · 1605-7422/07/\$18 · doi: 10.1117/12.702600 
field in optically scattering media that have a heterogeneous distribution of optical parameters. In addition, based on the theoretical models, Monte Carlo algorithms were developed and used for comparison with the theoretical predictions ${ }^{11,13-15,20}$ as well as for modeling the scattering samples that have optically absorbing objects of cylindrical shapes. ${ }^{21}$

In this paper, based on the ladder diagram approximation of the Bethe-Salpeter equation, ${ }^{22}$ we have derived a temporal correlation transfer equation (CTE) for multiply scattered light modulated by an ultrasonic pulse. The derivation is similar to the previous development of the CTE when CW ultrasound was used. ${ }^{15}$ In Sect. 3, we develop a Monte Carlo simulation, based on the CTE, which can be used to calculate the time-varying power spectral density of light modulated by pulsed and focused ultrasound in optically turbid media with heterogeneous distributions of optical parameters. We further calculate the time-varying spatial distribution of the power spectral density of the ultrasound-modulated light produced by an ultrasound pulse with a center frequency of $1-\mathrm{MHz}$ that is focused in an optically scattering slab. The scattering slab has two embedded optically absorbing objects positioned within the ultrasonic focal zone with background optical parameters representative of those in soft biological tissues at visible and near infrared wavelengths.

\section{DEVELOPMENT OF THE CTE}

The development of the CTE for multiply scattered light modulated by an ultrasound pulse is largely analogous to the derivation of the CTE for CW ultrasound, ${ }^{15}$ with the difference that a more general representation of the spatio-temporal dependence of ultrasound is used in the former case.

We first develop the approximate expressions for a Green's function $G_{a}\left(\mathbf{r}, \mathbf{r}_{0}, t\right)$ of the electric field component in a medium free from optical scatterers and absorbers in the presence of an ultrasound field, and for a mean Green's function $G_{s}\left(\mathbf{r}_{b}, \mathbf{r}_{a}, t\right)$ of the ensemble averaged electric field component in a medium having discrete and uncorrelated optical scatterers.

For moderate ultrasound pressures, the optical index of refraction experiences a small perturbation and we locally approximate it with $n(\mathbf{r}, t)=n_{0}\left[1+\eta P(\mathbf{r}, t) /\left(\rho v_{a}^{2}\right)\right]$, where $n_{0}$ is the unperturbed optical index of refraction; $P(\mathbf{r}, t)$ is the ultrasound pressure; $\rho$ is the mass density of the medium; $v_{a}$ is the ultrasound speed; and $\eta$ is the elasto-optical coefficient (in water at standard conditions $v_{a} \approx 1480 \mathrm{~ms}^{-1}$; and $\eta \approx 0.32$ ). We consider independent optical scattering in an optically scattering medium representative of soft biological tissue and assume the weak scattering approximation that the optical mean-free path $l_{s}$ is much greater than the optical wavelength $\lambda_{0}$. We also assume that the ultrasound field in volumes $\sim l_{t r}^{3}$ can be locally approximated as a plane wave $P(\mathbf{r}, t)=P_{0} h\left(t-v_{a} \hat{\mathbf{\Omega}}_{a} \cdot \mathbf{r}+\phi\right)$, where $h(x)$ is the propagation function of the ultrasound pulse normalized to unity, and $P_{0}, \phi$, and $\hat{\boldsymbol{\Omega}}_{a}$ are the local pressure amplitude, local initial phase, and local propagation direction unit vector of the ultrasound, respectively $\left(\left|\hat{\boldsymbol{\Omega}}_{a}\right|=1\right)$.

We consider a source of monochromatic light having angular frequency $\omega_{0}$ and wave-vector magnitude $k_{0}=$ $\omega_{0} / c_{0}$, where $c_{0}$ is the speed of light in vacuum. The time retardation and the optical polarization effects are neglected for simplicity and we consider only one component of the electric field vector $\tilde{E}(\mathbf{r}, t)$. Due to the large ratio of $\omega_{0}$ to the ultrasound angular frequency, we approximate the quasi-monochromatic electric field in the medium as $\tilde{E}(\mathbf{r}, t) \approx E(\mathbf{r}, t) \exp \left(-i \omega_{0} t\right)$, where $E(\mathbf{r}, t)$ is a slowly changing function of time. For a point source of light positioned at $\mathbf{r}_{0}$, the slowly changing amplitude $E(\mathbf{r}, t)$ is given by the Green's function $G_{a}\left(\mathbf{r}, \mathbf{r}_{0}, t\right)$ that can be expressed as

$$
G_{a}\left(\mathbf{r}, \mathbf{r}_{0}, t\right)=\frac{\exp \left(i k_{0} n_{0}\left|\mathbf{r}-\mathbf{r}_{0}\right|\left[1+\xi\left(\mathbf{r}, \mathbf{r}_{0}, t\right)\right]\right)}{-4 \pi\left|\mathbf{r}-\mathbf{r}_{0}\right|}
$$

where $2 \eta|P(\mathbf{r}, t)| /\left(\rho v_{a}^{2}\right) \ll 1, \delta()$ is the Dirac delta function and $\xi\left(\mathbf{r}, \mathbf{r}_{0}, t\right)$ is the small fractional phase perturbation that slowly varies in time and depends on $P(\mathbf{r}, t)$. We approximate $\xi\left(\mathbf{r}, \mathbf{r}_{0}, t\right)$ as

$$
\xi\left(\mathbf{r}, \mathbf{r}_{0}, t\right)=\frac{1}{2} M \int_{\mathbf{r}_{0}}^{\mathbf{r}} h\left(t-v_{a} \hat{\mathbf{\Omega}}_{a} \cdot \mathbf{r}^{\prime}+\phi\right) \mathrm{d} r^{\prime},
$$


where $M=2 \eta P_{0} /\left(\rho v_{a}^{2}\left|\mathbf{r}-\mathbf{r}_{0}\right|\right)$. We assume moderate ultrasound pressures, distances $\mathbf{r}$ not far from the source position $\mathbf{r}_{0}$ such that $k_{0} n_{0}\left|\mathbf{r}-\mathbf{r}_{0}\right| \xi\left(\mathbf{r}, \mathbf{r}_{0}, t\right) \ll 1$, and that the ultrasound-induced refraction of the optical waves is negligible for the interaction length $\left|\mathbf{r}-\mathbf{r}_{0}\right|$. In further derivations, the expressions for $\xi\left(\mathbf{r}, \mathbf{r}_{0}, t\right)$ [Eq. (2)] and for $G_{a}\left(\mathbf{r}, \mathbf{r}_{0}, t\right)$ [Eq. (1)] are required to be approximately valid for $\left|\mathbf{r}-\mathbf{r}_{0}\right|$ on the order of a few $l_{t r}$, which is satisfied in soft biological tissues at visible and near-infrared optical wavelengths $\left(l_{t r} \approx 1 \mathrm{~mm}\right)$, for moderate ultrasound pressures $\left(P_{0} \leq 10^{5} \mathrm{~Pa}\right)$ and in the medical ultrasound frequency range. ${ }^{13}$

The optical extinction, scattering and absorption coefficients are defined as $\mu_{t}=\mu_{s}+\mu_{a}, \mu_{s}=\sigma_{s} \rho_{s}$, and $\mu_{a}=\sigma_{a} \rho_{s}$, respectively, where $\rho_{s}$ is the density of optical scatterers, $\sigma_{s}$ is the optical scattering cross section, and $\sigma_{a}$ is the optical absorption cross section. We assume that the optical scattering amplitude $f\left(\hat{\boldsymbol{\Omega}}_{\mathrm{sc}}, \hat{\boldsymbol{\Omega}}_{\text {inc }}\right)$ is a function of $\hat{\boldsymbol{\Omega}}_{\mathrm{sc}} \cdot \hat{\boldsymbol{\Omega}}_{\mathrm{inc}}$ only, where $\hat{\boldsymbol{\Omega}}_{\mathrm{inc}}$ and $\hat{\boldsymbol{\Omega}}_{\mathrm{sc}}$ are the directions of the incident and scattered waves, respectively. The scattering phase function $p\left(\hat{\boldsymbol{\Omega}}_{\mathrm{sc}}, \hat{\boldsymbol{\Omega}}_{\text {inc }}\right)$ is defined as $p\left(\hat{\boldsymbol{\Omega}}_{\mathrm{sc}}, \hat{\boldsymbol{\Omega}}_{\text {inc }}\right)=\sigma_{s}^{-1}\left|f\left(\hat{\boldsymbol{\Omega}}_{\mathrm{sc}}, \hat{\boldsymbol{\Omega}}_{\text {inc }}\right)\right|^{2}$, and it satisfies $\int_{4 \pi} p\left(\hat{\boldsymbol{\Omega}}_{\mathrm{sc}}, \hat{\boldsymbol{\Omega}}_{\text {inc }}\right) \mathrm{d} \Omega_{\mathrm{sc}}=1$. From the optical theorem we also have $\sigma_{s}+\sigma_{a}=4 \pi \operatorname{Im}\left[f\left(\hat{\boldsymbol{\Omega}}_{\mathrm{inc}}, \hat{\boldsymbol{\Omega}}_{\text {inc }}\right)\right] /\left(k_{0} n_{0}\right)$, where $\operatorname{Im}[]$ takes on the imaginary part.

The ensemble averaged value of the electric field at $\mathbf{r}_{b}$ emitted from a point source at $\mathbf{r}_{a}$ is referred to also as a mean or coherent field and it is provided by a mean Green's function $G_{s}\left(\mathbf{r}_{b}, \mathbf{r}_{a}, t\right)$. We assume sufficiently small optical scatterers and consider only the far field approximations of the scattered fields. $G_{s}\left(\mathbf{r}_{b}, \mathbf{r}_{a}, t\right)$ is obtained by solving the Dyson equation, ${ }^{16,17,22}$ whose far-field expression in the Bourret approximation is given by

$$
G_{s}\left(\mathbf{r}_{b}, \mathbf{r}_{a}, t\right)=G_{a}\left(\mathbf{r}_{b}, \mathbf{r}_{a}, t\right)-4 \pi \rho_{s} \int G_{a}\left(\mathbf{r}_{b}, \mathbf{r}_{s}, t\right) f\left(\hat{\boldsymbol{\Omega}}_{\mathrm{sb}}, \hat{\boldsymbol{\Omega}}_{\mathrm{as}}\right) \exp \left[i k_{0} n_{0} \mathbf{e}_{s}(t) \cdot\left(\hat{\boldsymbol{\Omega}}_{\mathrm{as}}-\hat{\mathbf{\Omega}}_{\mathrm{sb}}\right)\right] G_{s}\left(\mathbf{r}_{s}, \mathbf{r}_{a}, t\right) \mathrm{d} \mathbf{r}_{s}
$$

The exponential term on the right hand side of Eq. (3) accounts for the Doppler shift caused by the ultrasoundinduced movement of the scatterer, and the position of the scatterer at time $t$ is $\mathbf{r}_{s}+\mathbf{e}_{s}(t)$, where $\mathbf{r}_{s}$ is the resting position and $\mathbf{e}_{s}(t)$ is the small ultrasound-induced displacement. The refraction of the mean optical field that is due to the ultrasound is neglected and $\hat{\boldsymbol{\Omega}}_{\mathrm{as}}$ and $\hat{\boldsymbol{\Omega}}_{\mathrm{sb}}$ are unity vectors in directions $\mathbf{r}_{s}-\mathbf{r}_{a}$ and $\mathbf{r}_{b}-\mathbf{r}_{s}$, respectively.

Equation (3) can be solved by applying the method of stationary phase in a way similar to the derivation presented in the Appendix of Ref. 15. The mean Green's function is expressed as

$$
G_{s}\left(\mathbf{r}_{b}, \mathbf{r}_{a}, t\right)=-\exp \left[i K\left(\mathbf{r}_{b}, \mathbf{r}_{a}, t\right)\left|\mathbf{r}_{b}-\mathbf{r}_{a}\right|\right] /\left(4 \pi\left|\mathbf{r}_{b}-\mathbf{r}_{a}\right|\right)
$$

where $K\left(\mathbf{r}_{b}, \mathbf{r}_{a}, t\right)=k_{0} n_{0}\left[1+\xi\left(\mathbf{r}_{b}, \mathbf{r}_{a}, t\right)\right]+2 \pi \rho_{s} f(\hat{\boldsymbol{\Omega}}, \hat{\boldsymbol{\Omega}}) /\left(k_{0} n_{0}\right) . G_{s}\left(\mathbf{r}_{b}, \mathbf{r}_{a}, t\right)$ is expressed in Eq. (4) similarly as in Ref. 15 where CW ultrasound is considered. However, the expression for $\xi\left(\mathbf{r}_{b}, \mathbf{r}_{a}, t\right)$ in Ref. 15 is just a special case of the more general expression in Eq. (2). The term $\xi\left(\mathbf{r}_{b}, \mathbf{r}_{a}, t\right)$ in propagation constant $K\left(\mathbf{r}_{b}, \mathbf{r}_{a}, t\right)$ is related to the accumulated optical phase from $\mathbf{r}_{a}$ to $\mathbf{r}_{b}$, due to ultrasound-induced changes in the optical index of refraction, and the term $2 \pi \rho_{s} f(\hat{\boldsymbol{\Omega}}, \hat{\boldsymbol{\Omega}}) /\left(k_{0} n_{0}\right)$ accounts for the multiple wave scattering from $\mathbf{r}_{a}$ to $\mathbf{r}_{b}$. In the absence of optical scatterers $\left(\rho_{s}=0\right), G_{s}\left(\mathbf{r}_{b}, \mathbf{r}_{a}, t\right)$ reduces to $G_{a}\left(\mathbf{r}_{b}, \mathbf{r}_{a}, t\right)$.

In order to obtain the time-varying power spectral density of multiply scattered light at time $t$, we present the mutual coherence function of the electric field component as $\Gamma\left(\mathbf{r}_{a}, \mathbf{r}_{b}, t, \tau\right)=\left\langle E\left(\mathbf{r}_{a}, t-\tau / 2\right) E^{*}\left(\mathbf{r}_{b}, t+\tau / 2\right)\right\rangle$, where $\mathbf{r}_{a}$ and $\mathbf{r}_{b}$ are two closely spaced points relative to the optical mean-free path $l_{t}$, and \langle\rangle represents ensemble averaging. We further assume that $\Gamma\left(\mathbf{r}_{a}, \mathbf{r}_{b}, t, \tau\right)$ is a quasiuniform function, which under the weak-scattering approximation satisfies the ladder approximation of the Bethe-Salpeter equation ${ }^{16,17,19,22}$ for moving scatterers

$$
\Gamma\left(\mathbf{r}_{a}, \mathbf{r}_{b}, t, \tau\right)=\Gamma_{0}\left(\mathbf{r}_{a}, \mathbf{r}_{b}, t, \tau\right)+\iint v_{s^{\prime}}^{a}(t-\tau / 2) v_{s^{\prime \prime}}^{b^{*}}(t+\tau / 2) \Gamma\left(\mathbf{r}_{s^{\prime}}, \mathbf{r}_{s^{\prime \prime}}, t, \tau\right) \rho\left(\mathbf{r}_{s^{\prime}}, t-\tau / 2 ; \mathbf{r}_{s^{\prime \prime}}, t+\tau / 2\right) \mathrm{d} \mathbf{r}_{s^{\prime}} \mathrm{d} \mathbf{r}_{s^{\prime \prime}}
$$

where $\mathbf{r}_{s^{\prime}}$ and $\mathbf{r}_{s^{\prime \prime}}$ are the positions of the same scatterer at times $t-\tau / 2$ and $t+\tau / 2$, respectively, and $\rho\left(\mathbf{r}_{s^{\prime}}, t-\tau / 2 ; \mathbf{r}_{s^{\prime \prime}}, t+\tau / 2\right)$ is the probability density of finding the same scatterer $s$ at position $\mathbf{r}_{s^{\prime}}$ and time $t-\tau / 2$, and at position $\mathbf{r}_{s^{\prime \prime}}$ and time $t+\tau / 2 . \Gamma_{0}\left(\mathbf{r}_{a}, \mathbf{r}_{b}, t, \tau\right)=\left\langle E\left(\mathbf{r}_{a}, t-\tau / 2\right)\right\rangle\left\langle E^{*}\left(\mathbf{r}_{b}, t+\tau / 2\right)\right\rangle$ is the mutual coherence function of the coherent (unscattered) field. 
We define the spectral density $\tilde{\Gamma}\left(\mathbf{r}_{c s}, \mathbf{q}^{\prime}, t, \tau\right)$ of $\Gamma\left(\mathbf{r}_{s^{\prime}}, \mathbf{r}_{s^{\prime \prime}}, t, \tau\right)$ as

$$
\tilde{\Gamma}\left(\mathbf{r}_{c s}, \mathbf{q}^{\prime}, t, \tau\right)=(2 \pi)^{-3} \int \Gamma\left(\mathbf{r}_{c s}, \mathbf{r}_{d s}, t, \tau\right) \exp \left(-i \mathbf{q}^{\prime} \cdot \mathbf{r}_{d s}\right) \mathrm{d} \mathbf{r}_{d s}
$$

where $\mathbf{r}_{d s}=\mathbf{r}_{s^{\prime}}-\mathbf{r}_{s^{\prime \prime}}$ and $\mathbf{r}_{c s}=\left(\mathbf{r}_{s^{\prime}}+\mathbf{r}_{s^{\prime \prime}}\right) / 2$ and we define vectors $\mathbf{r}_{c}=\left(\mathbf{r}_{a}+\mathbf{r}_{b}\right) / 2, \mathbf{r}_{d}=\mathbf{r}_{a}-\mathbf{r}_{b}$, and $\hat{\mathbf{\Omega}}=\left(\mathbf{r}_{c}-\mathbf{r}_{c s}\right) /\left|\mathbf{r}_{c}-\mathbf{r}_{c s}\right|$ in the center-of-gravity coordinate systems.

By following the derivation presented in Ref. 15 we obtain the integral form of the CTE as

$$
I(\mathbf{r}, \hat{\boldsymbol{\Omega}}, t, \tau)=I_{0}(\mathbf{r}, \hat{\boldsymbol{\Omega}}, t, \tau)+\int \mu_{s} p\left(\hat{\mathbf{\Omega}}, \hat{\boldsymbol{\Omega}}^{\prime}\right) \exp \left(-\mu_{t}\left|\mathbf{r}-\mathbf{r}_{s}\right|\right) I\left(\mathbf{r}_{s}, \hat{\mathbf{\Omega}}^{\prime}, t, \tau\right) \Phi\left(\mathbf{r}, \mathbf{r}_{s}, \hat{\mathbf{\Omega}}, \hat{\boldsymbol{\Omega}}^{\prime}, t, \tau\right) d\left|\mathbf{r}-\mathbf{r}_{s}\right| \mathrm{d} \Omega^{\prime},
$$

where $I(\mathbf{r}, \hat{\mathbf{\Omega}}, t, \tau)$ is the time-varying specific intensity given by

$$
\Gamma\left(\mathbf{r}_{c}, \mathbf{r}_{d}, t, \tau\right)=\int I\left(\mathbf{r}_{c}, \hat{\mathbf{\Omega}}, t, \tau\right) \exp \left(i K_{r} \hat{\mathbf{\Omega}} \cdot \mathbf{r}_{d}\right) \mathrm{d} \Omega
$$

In Eq. 7, the factor $\Phi\left(\mathbf{r}, \mathbf{r}_{s}, \hat{\boldsymbol{\Omega}}, \hat{\mathbf{\Omega}}^{\prime}, t, \tau\right)=\exp \left[i \Psi_{d}\left(\mathbf{r}_{s}, \hat{\boldsymbol{\Omega}}, \hat{\mathbf{\Omega}}^{\prime}, t, \tau\right)\right] \exp \left[i \Psi_{n}\left(\mathbf{r}, \mathbf{r}_{s}, t, \tau\right)\right]$ accounts for the ultrasound-induced optical phase increments due to both mechanisms of modulation. The term $\Psi_{n}\left(\mathbf{r}_{c}, \mathbf{r}_{c s}, t, \tau\right)$ is the difference between the optical phase increments that are due to ultrasound-induced optical index of refraction changes and it is given by

$$
\Psi_{n}\left(\mathbf{r}_{c}, \mathbf{r}_{c s}, t, \tau\right)=\frac{1}{2} k_{0} n_{0} M \int_{\mathbf{r}_{c s}}^{\mathbf{r}_{c}}\left[h\left(t-\tau / 2-v_{a} \hat{\boldsymbol{\Omega}}_{a} \cdot \mathbf{r}^{\prime}+\phi\right)-h\left(t+\tau / 2-v_{a} \hat{\mathbf{\Omega}}_{a} \cdot \mathbf{r}^{\prime}+\phi\right)\right] \mathrm{d} r^{\prime} .
$$
by

The term $\Psi_{d}\left(\mathbf{r}_{s}, \hat{\boldsymbol{\Omega}}, \hat{\boldsymbol{\Omega}}^{\prime}, t, \tau\right)$ is due to ultrasound-induced displacement of the optical scatterers and it is given

$$
\Psi_{d}\left(\mathbf{r}_{s}, \hat{\mathbf{\Omega}}, \hat{\mathbf{\Omega}}^{\prime}, t, \tau\right)=\frac{K_{r} P_{0}}{\rho v_{a}}\left[\left(\hat{\mathbf{\Omega}}-\hat{\mathbf{\Omega}}^{\prime}\right) \cdot \hat{\mathbf{\Omega}}_{a}\right] \int_{t-\tau / 2}^{t+\tau / 2} h\left(t^{\prime}-v_{a} \hat{\mathbf{\Omega}}_{a} \cdot \mathbf{r}_{s}+\phi\right) \mathrm{d} t^{\prime}
$$

where $K_{r}=n_{0} k_{0}+4 \pi \operatorname{Re}[f(\hat{\boldsymbol{\Omega}}, \hat{\boldsymbol{\Omega}})] \rho_{s} /\left(2 k_{0} n_{0}\right), \operatorname{Re}[]$ takes on the real part and we assume that optical scatterers follow the ultrasound-induced fluid movement in amplitude and phase. ${ }^{13}$

We see that the time-varying specific intensity $I(\mathbf{r}, \hat{\boldsymbol{\Omega}}, t, \tau)$ in Eq. (7) is given as a sum of all time-varying specific intensities scattered into direction $\hat{\boldsymbol{\Omega}}$ including the unscattered term $I_{0}(\mathbf{r}, \hat{\boldsymbol{\Omega}}, t, \tau)$. Similarly to the previously derived CTE for moving scatterers undergoing Brownian motion, ${ }^{16,19}$ in Eq. (7) we have a term $\Psi_{d}()$ that is due to the ultrasound-induced movement of the optical scatterers. In addition, in Eq. (7) we also have a new term $\Psi_{n}()$ that is due to ultrasound-induced changes in the optical index of refraction.

We can further use the time-varying specific intensity $I\left(\mathbf{r}_{c}, \hat{\mathbf{\Omega}}, t, \tau\right)$ to obtain the time-varying optical power spectral density $P\left(\mathbf{r}_{c}, t, \omega\right)$ of the ultrasound modulated light received in some solid angle $\Omega_{0}$ based on the Wigner distribution $^{29}$ as

$$
P\left(\mathbf{r}_{c}, t, \omega\right)=\frac{1}{2 \pi} \int_{-\infty}^{+\infty} I_{\Omega_{0}}\left(\mathbf{r}_{c}, t, \tau\right) \exp (i \omega t) \mathrm{d} t
$$

where $I_{\Omega_{0}}\left(\mathbf{r}_{c}, t, \tau\right)=\int_{\Omega_{0}} I\left(\mathbf{r}_{c}, \hat{\mathbf{\Omega}}, t, \tau\right) \mathrm{d} \Omega$.

Analytical solutions for $I(\mathbf{r}, \hat{\boldsymbol{\Omega}}, t, \tau)$ and $P(\mathbf{r}, t, \omega)$ are difficult to find from Eqs. (7) and (11) for any practical experimental configurations. Numerical codes and Monte Carlo algorithms, however, can be readily developed. In Sect. 3, one such Monte Carlo algorithm is presented. 


\section{MONTE CARLO SIMULATION}

We developed a Monte Carlo (MC) algorithm, which can be used to calculate the time-varying power spectral density of light modulated by a pulsed ultrasound field focused in an optically scattering medium with a heterogeneous distribution of optical parameters. We first divide the optically scattering medium into cells along the Cartesian axes and assign a vector $\mathbf{n}$ with integer coordinates $\left\{n_{x}, n_{y}, n_{z}\right\}$ to each cell. Next, we assign values of the optical absorption $\left(\mu_{a, \mathbf{n}}\right)$ and the scattering $\left(\mu_{s, \mathbf{n}}\right)$ coefficients as well as the scattering anisotropy factor $\left(g_{\mathbf{n}}\right)$ to each cell, where a Henyey-Greenstein scattering phase function is assumed. ${ }^{30}$ The local parameters of the ultrasound field are also assigned to each cell: an average ultrasound propagation direction $\hat{\boldsymbol{\Omega}}_{a, \mathbf{n}}$, pressure amplitude $P_{0, \mathbf{n}}$, and time delay of the pulse $t_{\mathbf{n}}$. We assume that the dimensions of each cell are much smaller than the ultrasound wavelength and that the ultrasound field can be approximated within cell as $P_{\mathbf{n}}(t)=P_{0, \mathbf{n}} h\left(t-t_{\mathbf{n}}\right)$, where $h(t)$ is the propagation function of the ultrasound pulse normalized to unity. We also assume that the error due to using the same propagation function $h(t)$ in all the cells is small. The procedure for propagation of the photon packets in the MC is the same as in Ref. 15 , and similar to the previously described algorithms. ${ }^{20,31}$ The photon packet is analyzed at each crossing of the cell boundaries, and the remaining length of the free path is adjusted based on the extinction coefficient within the cell that the photon packet is entering.

The trajectory of each photon consists of many small steps, which are determined by all of the scattering events and cell boundaries along the way. For each small photon step of length $l_{k}$ within cell $\mathbf{m}_{k}$, we calculate the optical phase increment due to ultrasound-induced index of refraction changes as $\Delta \varphi_{n, k}(t)=k_{0} n_{0} l_{k} P_{\mathbf{m}_{k}}(t) \eta /\left(\rho v_{a}^{2}\right)$. Similarly, for each scattering event $j$ within cell $\mathbf{n}_{j}$, we calculate the optical phase increment that is due to ultrasound-induced scatterer displacement as $\Delta \varphi_{d, j}(t)=k_{0} n_{0}\left[\left(\hat{\boldsymbol{\Omega}}_{\mathrm{inc}, j}-\hat{\boldsymbol{\Omega}}_{\mathrm{sc}, j}\right) \cdot \hat{\boldsymbol{\Omega}}_{a, \mathbf{n}_{j}}\right] A_{\mathbf{n}_{j}}(t)$, where $\hat{\boldsymbol{\Omega}}_{a, \mathbf{n}_{j}} A_{\mathbf{n}_{j}}(t)$ is the scatterer displacement vector, and $\hat{\boldsymbol{\Omega}}_{\mathrm{inc}, j}$ and $\hat{\boldsymbol{\Omega}}_{\mathrm{sc}, j}$ are the incident and scattered photon directions, respectively.

At each scattering event, the total ultrasound-induced phase increment of the photon packet accumulated up to this point is $\Delta \varphi(t)=\sum_{k} \Delta \varphi_{n, k}(t)+\sum_{j} \Delta \varphi_{d, j}(t)$, and $k$ and $j$ count all of the previous steps and scattering events of the photon. We calculate the time-varying power spectral density $W_{p h} P(t, \omega)$ for the photon packet at time $t$ based on the Wigner distribution ${ }^{29}$ as

$$
W_{p h} P(t, \omega)=W_{p h}(2 \pi)^{-1} \int_{-\infty}^{+\infty} W(t, \tau) \exp (i \omega \tau) \mathrm{d} \tau,
$$

where $W_{p h}$ is current weight of a photon, $P(t, \omega)$ is normalized time-varying power spectral density, $W(t, \tau)=$ $\exp [i \Delta \varphi(t, \tau)]$ and $\Delta \varphi(t, \tau)=\Delta \varphi(t+\tau / 2)-\Delta \varphi(t-\tau / 2)$. In Eq. (12), $\omega$ represents the angular frequency increment in respect to the angular frequency $\omega_{0}$ of the non-modulated light.

We further assume that the difference in phase increment $\Delta \varphi(t, \tau)$ is small $(|\Delta \varphi(t, \tau)| \ll 1)$, and consider only the first three terms in the Taylor expansion of $W(t, \tau)$. The approximate expression for $P(t, \omega)$ from Eq. $(12)$ is then given by

$$
P(t, \omega) \approx(2 \pi)^{-1} \int_{-\infty}^{+\infty}\left[1+i \Delta \varphi(t, \tau)-\frac{1}{2} \Delta \varphi^{2}(t, \tau)\right] \exp (i \omega \tau) \mathrm{d} \tau
$$

We proceed by integrating the individual terms in Eq. (13). The phase increment difference $\Delta \varphi(t, \tau)$ is given by

$$
\begin{aligned}
\Delta \varphi(t, \tau)= & \frac{k_{0} n_{0} \eta}{\rho v_{a}^{2}} \sum_{k} l_{k} P_{0, \mathbf{m}_{k}}\left[h\left(t+\tau / 2-t_{\mathbf{m}_{k}}\right)-h\left(t-\tau / 2-t_{\mathbf{m}_{k}}\right)\right] \\
& +\frac{k_{0} n_{0}}{\rho v_{a}} \sum_{j}\left[\left(\hat{\boldsymbol{\Omega}}_{\mathrm{inc}, j}-\hat{\boldsymbol{\Omega}}_{\mathrm{sc}, j}\right) \cdot \hat{\boldsymbol{\Omega}}_{a, \mathbf{n}_{j}}\right] P_{0, \mathbf{n}_{j}} \int_{t-\tau / 2}^{t+\tau / 2} h\left(u-t_{\mathbf{n}_{j}}\right) \mathrm{d} u
\end{aligned}
$$

where we assumed that optical scatterers follows the ultrasound-induced fluid oscillations in amplitude and phase such that $\left(\rho v_{a}\right)^{-1} P_{0, \mathbf{n}_{j}} \int_{t-\tau / 2}^{t+\tau / 2} h\left(u-t_{\mathbf{n}_{j}}\right) \mathrm{d} u=A_{\mathbf{n}_{j}}(t+\tau / 2)-A_{\mathbf{n}_{j}}(t-\tau / 2)$. 
We denote with $P_{0}(t, \omega)$ the integral of the first term $\left[(2 \pi)^{-1} \int \exp (i \omega \tau) \mathrm{d} \tau \equiv \delta(\omega)\right]$ in Eq. (13). Thus, $W_{p h} P_{0}(t, \omega)$ contributes a value of $W_{p h}$ to the unmodulated intensity of light at time $t$.

The integral of the second term in Eq. (13) $\left[(2 \pi)^{-1} \int_{-\infty}^{+\infty} i \Delta \varphi(t, \tau) \exp (i \omega \tau) \mathrm{d} \tau\right]$ is then denoted with $P_{1}(t, \omega)$ and expressed as

$$
\begin{aligned}
P_{1}(t, \omega)= & -\frac{4 k_{0} n_{0} \eta}{\rho v_{a}^{2}} \sum_{k} l_{k} P_{0, \mathbf{m}_{k}} \operatorname{Im}\left[\tilde{h}(2 \omega) \exp \left[-i 2 \omega\left(t-t_{\mathbf{m}_{k}}\right)\right]\right] \\
& -\frac{4 k_{0} n_{0}}{\rho v_{a}} \sum_{j} P_{0, \mathbf{n}_{j}}\left[\left(\hat{\boldsymbol{\Omega}}_{\mathrm{inc}, j}-\hat{\boldsymbol{\Omega}}_{\mathrm{sc}, j}\right) \cdot \hat{\boldsymbol{\Omega}}_{a, \mathbf{n}_{j}}\right] \operatorname{Re}\left[\frac{\tilde{h}(2 \omega)}{2 \omega} \exp \left[-i 2 \omega\left(t-t_{\mathbf{n}_{j}}\right)\right]\right],
\end{aligned}
$$

where $\tilde{h}(\omega)$ is the Fourier transform of $h(t)$. It is physically meaningful to require that there is no dc component in the temporal spectrum of the ultrasonic pulse $(\tilde{h}(0)=0)$, which ensures that expression for $P_{1}(t, \omega)$ in Eq. $(15)$ is well defined for all angular frequencies $\omega$ and that $P_{1}(t, 0)=0$. Owing to the factor $\exp (2 i \omega t)$ in Eq. (13), $P_{1}(t, \omega)$ fluctuates fast in time. If $T_{a}$ is the time period associated with the central frequency of the ultrasound pulse, then the average value of $P_{1}(t, \omega)$ is approximately zero in any time interval that is comparable or longer than $T_{a} / 2$ and we will neglect it in further derivations.

Finally, the more complex expression for the integral of the third term in Eq. (13) is given by

$$
P_{2}(t, \omega)=-\frac{1}{2} \frac{1}{2 \pi} \int_{-\infty}^{+\infty} \Delta \varphi^{2}(t, \tau) \exp (i \omega \tau) \mathrm{d} \tau
$$

Since $\Delta \varphi(t, \tau)=\sum_{k} \Delta \varphi_{n, k}(t, \tau)+\sum_{j} \Delta \varphi_{d, j}(t, \tau)$, where $\Delta \varphi_{n, k}(t, \tau)=\Delta \varphi_{n, k}(t+\tau / 2)-\Delta \varphi_{n, k}(t-\tau / 2)$ and $\Delta \varphi_{d, j}(t, \tau)=\Delta \varphi_{d, j}(t+\tau / 2)-\Delta \varphi_{d, j}(t-\tau / 2)$, we express $P_{2}(t, \omega)$ as

$$
P_{2}(t, \omega)=P_{2, n n}(t, \omega)+P_{2, d d}(t, \omega)+2 P_{2, n d}(t, \omega),
$$

where terms $P_{2, a b}(t, \omega)$ in Eq. (17) are given by

$$
P_{2, a b}(t, \omega)=-\frac{1}{2} \frac{1}{2 \pi} \int_{-\infty}^{+\infty} \sum_{k, j} \Delta \varphi_{a, k}(t, \tau) \Delta \varphi_{b, j}(t, \tau) \exp (i \omega \tau) \mathrm{d} \tau .
$$

The difficulty of obtaining analytical solutions for $P_{2, a b}(t, \omega)$ depends on the particular form of the pulse propagation function $h(t)$. We choose a convenient but still general form of $h(t)$ as $h(t)=\omega_{a}^{-1} \partial h_{0}(t) / \partial t$, where $h_{0}(t)=\exp \left[-t^{2} /\left(2 \sigma^{2} T_{a}^{2}\right)\right] \sin \left(\omega_{a} t\right)$ is a simple periodic function bounded with a Gaussian envelope. Here, $T_{a}$ and $\omega_{a}=2 \pi / T_{a}$ are the temporal period and angular frequency of the sound wave associated with the central frequency of the pulse, respectively, and $\sigma$ is a nondimensional parameter that is used to control the temporal width of the pulse. The function $h(t)$ and its Fourier transform $\tilde{h}(\omega)$ are given by

$$
\begin{gathered}
h(t)=\exp \left(-\frac{t^{2}}{2\left(\sigma T_{a}\right)^{2}}\right)\left[\cos \left(\omega_{a} t\right)-\frac{t}{\omega_{a}\left(\sigma T_{a}\right)^{2}} \sin \left(\omega_{a} t\right)\right], \\
\tilde{h}(\omega)=\frac{1}{2} \frac{\omega}{\omega_{a} \sigma_{a} \sqrt{2 \pi}}\left[\exp \left(-\frac{\left(\omega+\omega_{a}\right)^{2}}{2 \sigma_{a}^{2}}\right)-\exp \left(-\frac{\left(\omega-\omega_{a}\right)^{2}}{2 \sigma_{a}^{2}}\right)\right],
\end{gathered}
$$

where $\sigma_{a}=1 /\left(\sigma T_{a}\right)$.

The approximate values of the integrals $I_{a b, k, j}(t, \omega)=(2 \pi)^{-1} \int \Delta \varphi_{a, k}(t, \tau) \Delta \varphi_{b, j}(t, \tau) \exp (i \omega \tau) \mathrm{d} \tau$ of individual terms in Eq. (18) can be expressed as

$$
I_{n n, k, j}(t, \omega)=-\Lambda \eta^{2} v_{a}^{-2} l_{k} l_{j} P_{0, \mathbf{m}_{k}} P_{0, \mathbf{n}_{j}} \omega^{2} \Psi\left(t, t_{\mathbf{m}_{k}}, t_{\mathbf{n}_{j}}\right) \Xi(\omega) \cos \left[\omega\left(t_{\mathbf{m}_{k}}-t_{\mathbf{n}_{j}}\right)\right],
$$




$$
\begin{gathered}
I_{d d, k, j}(t, \omega)=- \\
\quad \Lambda P_{0, \mathbf{m}_{k}} P_{0, \mathbf{n}_{j}}\left[\hat{\mathbf{\Omega}}_{a, \mathbf{m}_{k}} \cdot\left(\hat{\boldsymbol{\Omega}}_{\mathrm{inc}, k}-\hat{\mathbf{\Omega}}_{\mathrm{sc}, k}\right)\right]\left[\hat{\boldsymbol{\Omega}}_{a, \mathbf{n}_{j}} \cdot\left(\hat{\boldsymbol{\Omega}}_{\mathrm{inc}, j}-\hat{\mathbf{\Omega}}_{\mathrm{sc}, j}\right)\right] \\
\quad \times \Psi\left(t, t_{\mathbf{m}_{k}}, t_{\mathbf{n}_{j}}\right) \Xi(\omega) \cos \left[\omega\left(t_{\mathbf{m}_{k}}-t_{\mathbf{n}_{j}}\right)\right], \\
I_{n d, k, j}(t, \omega)=-\Lambda \eta v_{a}^{-1} l_{k} P_{0, \mathbf{m}_{k}} P_{0, \mathbf{n}_{j}}\left[\hat{\mathbf{\Omega}}_{a, \mathbf{n}_{j}} \cdot\left(\hat{\boldsymbol{\Omega}}_{\mathrm{inc}, j}-\hat{\mathbf{\Omega}}_{\mathrm{sc}, j}\right)\right] \\
\times \Psi\left(t, t_{\mathbf{m}_{k}}, t_{\mathbf{n}_{j}}\right) \Xi(\omega)\left(\omega \sin \left[\omega\left(t_{\mathbf{m}_{k}}-t_{\mathbf{n}_{j}}\right)\right]-\sigma_{a}^{2}\left(t-\frac{t_{\mathbf{m}_{k}}+t_{\mathbf{n}_{j}}}{2}\right) \cos \left[\omega\left(t_{\mathbf{m}_{k}}-t_{\mathbf{n}_{j}}\right)\right]\right),
\end{gathered}
$$

where $\Lambda=\left(k_{0} n_{0}\right)^{2} /\left[2\left(\rho v_{a}\right)^{2} \omega_{a}^{2} \sigma_{a} \sqrt{\pi}\right], \Psi\left(t, t_{\mathbf{m}_{k}}, t_{\mathbf{n}_{j}}\right)=\exp \left[-\sigma_{a}^{2}\left(t-t_{\mathbf{m}_{k}} / 2-t_{\mathbf{n}_{j}} / 2\right)^{2}\right], \Xi(\omega)=\exp \left[-\left(\omega+\omega_{a}\right)^{2} / \sigma_{a}^{2}\right]+$ $\exp \left[-\left(\omega-\omega_{a}\right)^{2} / \sigma_{a}^{2}\right]$, and we neglected the terms containing fast fluctuating factors $\exp \left[-2 i \omega\left(2 t-t_{\mathbf{m}_{k}}-t_{\mathbf{n}_{j}}\right)\right]$.

Let $M$ and $N_{M}$ represent the total number of scattering events and the total number of free steps, respectively, of the photon at scattering event $M$ that happens at time $t$. Since we analyze the photon propagation at each cell boundary, the number of small free steps $N_{M}$ can be greater than $M$. We calculate the value of $P_{2}(t, \omega)$ associated with the photon at each scattering event $M$ as

$$
P_{2}(t, \omega)=-\frac{1}{2} \sum_{k=1}^{N_{M}} \sum_{j=1}^{N_{M}} I_{n n, k, j}(t, \omega)-\frac{1}{2} \sum_{k=1}^{M} \sum_{j=1}^{M} I_{d d, k, j}(t, \omega)-\sum_{k=1}^{N_{M}} \sum_{j=1}^{M} I_{n d, k, j}(t, \omega) .
$$

We specify a discrete set of times $t_{s}$ during the time of flight of the ultrasonic pulse in the scattering sample, and a discrete set of angular frequencies $\omega_{q}$ around the central angular frequency of the ultrasound pulse $\omega_{a}$. At every scattering event of the photon, quantity $P_{2}\left(t_{s}, \omega_{q}\right)$ is calculated for all $t_{s}$ and $\omega_{q}$, and $\Delta P_{2, \mathbf{m}}\left(t_{s}, \omega_{q}\right)=P_{2}\left(t_{s}, \omega_{q}\right) \Delta W_{p h}$ is evaluated, where $\Delta W_{p h}=W_{p h} \mu_{a, \mathbf{m}} / \mu_{t, \mathbf{m}}$, and $W_{p h}$ is the current weight of a photon at the scattering event that occurs in cell $\mathbf{m}$. At the end of the simulation of all of the photon packets, sums $P_{2, \mathbf{m}}\left(t_{s}, \omega_{q}\right)=\sum \Delta P_{2, \mathbf{m}}\left(t_{s}, \omega_{q}\right)$ of the increments for all of the scattering events that have happened in cell $\mathbf{m}$ are proportional to the time-varying power spectral density of light at time $t_{s}$ and angular frequency $\omega_{q}$.

The sample in our simulation is an optically scattering slab with a thickness of $20 \mathrm{~mm}$ along the $X$ axis. The dimensions of the slab in the $Y$ and $Z$ directions are both $100 \mathrm{~mm}$, which minimizes the error of the simulation within the central region. We define the background optical properties of the scattering slab as $\mu_{a}=0.1 \mathrm{~cm}^{-1}$ and $\mu_{s}=10 \mathrm{~cm}^{-1}$ in the entire slab, which are representative of soft biological tissue for visible and near-infrared light, and, for simplicity, assume isotropic scattering. An ultrasound pulse propagates parallelly to the $Z$ axis within the slab, spaced at equal distances from the slab surfaces. The temporal profile of the pulse is given by Eq. (19), with the central frequency of $1 \mathrm{MHz}$ and $\sigma=0.65$. The focal spot of the transducer coincides with the center of the slab $(\{x, y, z\}=\{10 \mathrm{~mm}, 0 \mathrm{~mm}, 0 \mathrm{~mm}\})$, and the peak pressure amplitude $P_{0}$ of the ultrasonic pulse at the focus is $10^{5} \mathrm{~Pa}$. In order to model the ultrasound field we define the concave transducer element with an aperture diameter of $25.4 \mathrm{~mm}$ and a focal length of $40 \mathrm{~mm}$. The distributions of the ultrasound pressure and phase are calculated with publicly available software Field $\mathrm{II}^{32}$ versus time $t_{s}$ equally spaced by $0.5 \mu \mathrm{s}$. The ultrasound propagation directions are subsequently obtained by taking the gradient of the ultrasound phase. A pencil light source with a wavelength of $532 \mathrm{~nm}$ irradiates the scattering slab from the $x<0$ half space, at position $\{x, y, z\}=\{0 \mathrm{~mm}, 0 \mathrm{~mm}, 0 \mathrm{~mm}\}$. We assume the same optical index of refraction $n_{0}=1.33$ in whole space, a mass density of the medium $\rho=10^{3} \mathrm{kgm}^{-3}$, an ultrasound velocity $v_{a}=1480 \mathrm{~ms}^{-1}$, and an elasto-optical coefficient of water at room temperature $\eta=0.32$. The dimensions of the cells that divide the simulation volume are $\Delta x=0.5 \mathrm{~mm}, \Delta y=0.5 \mathrm{~mm}$, and $\Delta z=0.1 \mathrm{~mm}$, such that the change in ultrasound phase within each cell is small. The $\Delta P_{2, \mathbf{m}}\left(t_{s}, \omega_{q}\right)$ values are sampled at discrete frequencies $\omega_{q}=2 \pi f_{q}$, where $f_{q}$ is equally spaced by $\Delta f_{q} \approx 82 \mathrm{kHz}$ from $265 \mathrm{kHz}$ to $1.74 \mathrm{MHz}$. In order to reduce the memory requirement, the values of $\Delta P_{2, \mathbf{m}}\left(t_{s}, \omega_{q}\right)$ are recorded only in cells $\mathbf{m}$ that belong to the plane defined by $y=0 \mathrm{~mm}$.

Next, we position two optically absorbing cylinders $\left(\mu_{a}=100 \mathrm{~cm}^{-1}\right)$, both $2 \mathrm{~mm}$ in diameter and $2 \mathrm{~mm}$ long, within the scattering sample. The axes of both cylinders are parallel to the propagation direction of the ultrasound pulse. The separation between the absorbing objects is $2 \mathrm{~mm}$ and the center of separation coincides with the focal point of the ultrasound pulse $(\{x, y, z\}=\{10 \mathrm{~mm}, 0 \mathrm{~mm}, 0 \mathrm{~mm}\})$. In Fig. 1, we show the timevarying power of the ultrasound-modulated light simulated for the duration of propagation of the ultrasound pulse 

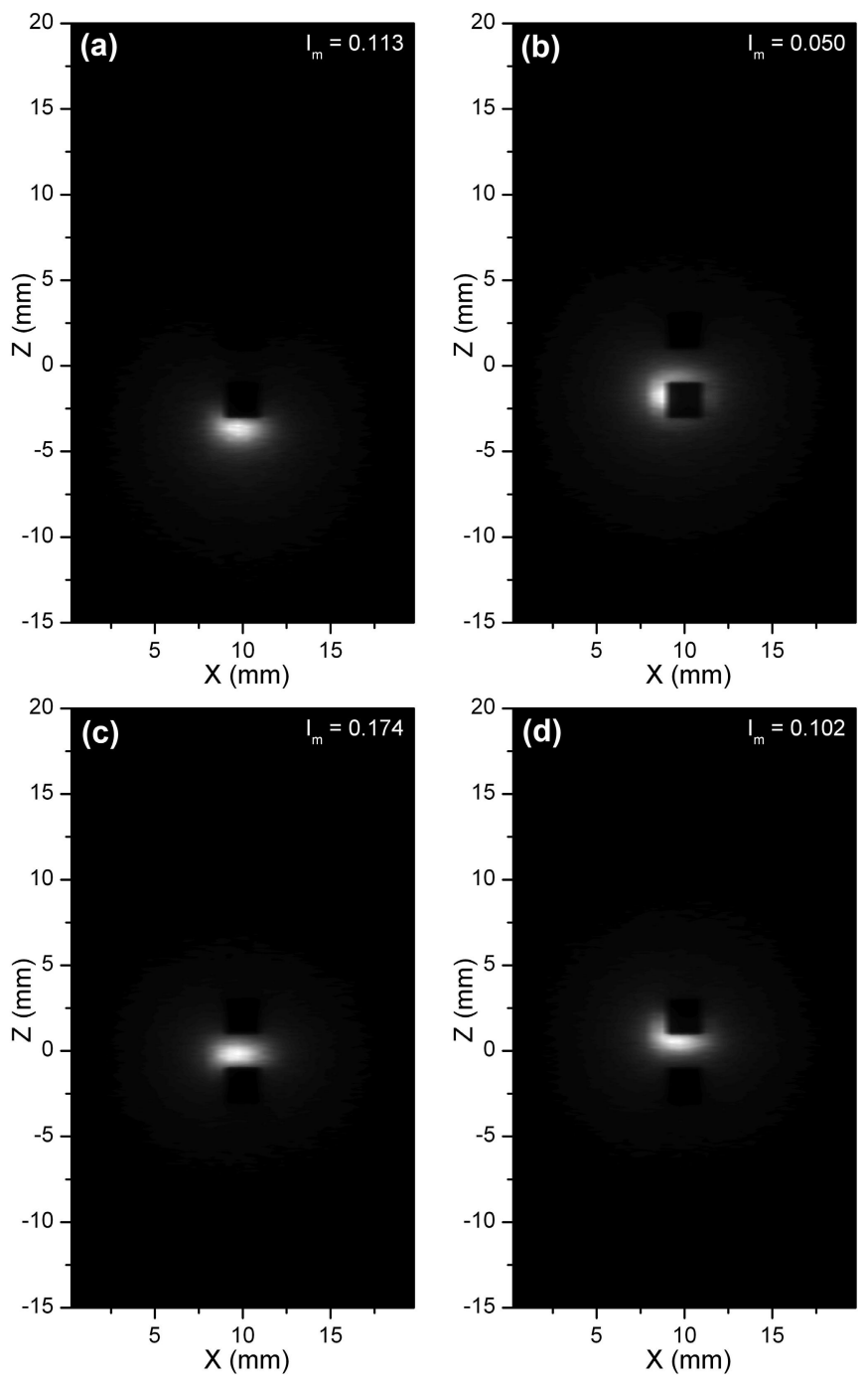

Figure 1. The time-varying power of the ultrasound-modulated light simulated for the duration of time-of-flight of the ultrasound pulse in the scattering sample. The spatial distribution of the power is presented in the plane defined by $y=0 \mathrm{~mm}$. The values are presented in shades of gray, with the levels equally spaced from zero to the maximum value. The maximum value of distribution $\left(I_{m}\right)$ in each frame is presented at the upper right corner.

in the scattering sample. The values of the time-varying power of the ultrasound-modulated light in each cell $\mathbf{m}$ are calculated from $P_{2, \mathbf{m}}\left(t_{s}, \omega_{q}\right)$ by multiplying with $2 \pi \Delta f_{q} \mu_{a, \mathbf{m}}$ the sum of all of the $\omega_{q}$ spectral components around the central frequency $\omega_{a}$ and by subsequently multiplying the obtained value with 2 to account for both first order sidebands of the modulated light. The consecutive frames in Fig. 1 are equally spaced by $1.0 \mu s$ time intervals, and in each frame the spatial distribution of the power is presented in the plane defined by $y=0 \mathrm{~mm}$. The values are presented in shades of gray, with the levels equally spaced from zero to the maximum value. The maximum value of distribution $\left(I_{m}\right)$ in each frame is presented at the upper right corner. The intensity of the ultrasound-modulated light is much higher when the ultrasound pulse is close to the focal point. The distribution of ultrasound-modulated light has sharp boundaries at the edges of absorbing objects. In addition, overlap of the ultrasound pulse with the position of either one of the absorbing objects causes a very significant decay in the intensity of ultrasound-modulated light that is generated. 


\section{CONCLUSION}

In conclusion, based on the ladder approximation of the Bethe-Salpeter equation and Wigner time-frequency distribution, we have developed an integral form of the CTE for multiply scattered light modulated by an ultrasound pulse. The derivations are valid under the weak-scattering approximation within the medical ultrasound frequency range and under moderate ultrasound pressures, and further theoretical development should address the setups with highly focused ultrasound and with very high ultrasound pressure. We have also developed a Monte Carlo algorithm that can be used to calculate the time-varying power spectral density of light modulated by the focused ultrasound pulse in optically turbid media with heterogeneous distributions of optical parameters. This permitted us, for the first time, to obtain the spatial distribution of the ultrasound-modulated light intensity during the ultrasound pulse propagation in the scattering sample that contains optically absorbing objects. We expect the CTE and MC to be of use for the estimation of resolution, contrast, and signal-to-noise ratios in UOT.

\section{Acknowledgment}

This project was sponsored by NIH Grant No. R33 CA094267.

\section{REFERENCES}

1. F. A. Marks, H. W. Tomlinson, and G. W. Brooksby, "A comprehensive approach to brest cancer detection using light: photon localization by ultrasound modulation and tissue characterization by spectral discrimination," in Proc. SPIE, 1888, pp. 500-510, 1993.

2. L. V. Wang, S. L. Jacques, and X. Zhao, "Continuous-wave ultrasonic modulation of scattered laser light to image objects in turbid media," Opt. Lett. 20, pp. 629-631, 1995.

3. S. Sakadzic and L. V. Wang, "High-resolution ultrasound-modulated optical tomography in biological tissues," Opt. Lett 29, pp. 2770-2772, Dec 2004.

4. C. Kim, R. J. Zemp, and L. V. Wang, "Intense acoustic bursts as a signal-enhancement mechanism in ultrasound-modulated optical tomography," Opt. Lett. 31, pp. 2423-2425, 2006.

5. W. Leutz and G. Maret, "Ultrasonic modulation of multiply scattered-light," Physica B 204, pp. 14-19, 1995.

6. M. Kempe, M. Larionov, D. Zaslavsky, and A. Z. Genack, "Acousto-optic tomography with multiply scattered light," J. Opt. Soc. Am. A 14, pp. 1151-1158, 1997.

7. G. D. Mahan, W. E. Engler, J. J. Tiemann, and E. G. Uzgiris, "Ultrasonic tagging of light: Theory," in Proc. Natl. Acad. Sci. USA, 95, p. 14015, 1998.

8. L. V. Wang, "Mechanisms of ultrasonic modulation of multiply scattered coherent light: an analytic model," Phys. Rev. Lett. 87, pp. 043903-(1-4), 2001.

9. G. Maret and P. E. Wolf, "Multiple light-scattering from disordered media - the effect of brownian-motion of scatterers," Z. Phys. B 65, pp. 409-413, 1987.

10. D. J. Pine, D. A. Weitz, P. M. Chaikin, and E. Herbolzheimer, "Diffusing-wave spectroscopy," Phys. Rev. Lett. 60, pp. 1134-1137, 1988.

11. S. Sakadzic and L. V. Wang, "Ultrasonic modulation of multiply scattered coherent light: an analytical model for anisotropically scattering media," Phys. Rev. E 66, pp. 026603-(19), 2002.

12. A. Lev and B. Sfez, "In vivo demonstration of the ultrasound modulated light technique," J. Opt. Soc. Am. A 20, pp. 2347-2354, 2003.

13. S. Sakadzic and L. V. Wang, "Modulation of multiply scattered coherent light by ultrasonic pulses: An analytical model," Phys. Rev. E 72, pp. 036620-(1-12), 2005.

14. S. Sakadzic and L. V. Wang, "Correlation transfer and diffusion of ultrasound-modulated multiply scattered light," Phys. Rev. Lett. 96, pp. 163902-(14), 2006.

15. S. Sakadzic and L. V. Wang, "Correlation transfer equation for ultrasound-modulated multiply scattered light," Phys. Rev. E 74, pp. 036618-(110), 2006.

16. A. Ishimaru, "Correlation functions of a wave in a random distribution of stationary and moving scatterers," Radio Sci. 10, pp. 45-52, 1975. 
17. M. J. Stephen, "Temporal fluctuations in wave propagation in random media," Phys. Rev. B 37, pp. 1-5, 1988.

18. F. C. MacKintosh and J. Sajeev, "Diffusing-wave spectroscopy and multiple scattering of light in correlated random media," Phys. Rev. B 40, pp. 2383-2406, 1989.

19. R. L. Dougherty, B. J. Ackerson, N. M. Reguigui, F. Dorri-Nowkoorani, and U. Nobbmann, "Correlation transfer: development and application," J. Quant. Spectrosc. Radiat. Transfer 52, pp. 713-727, 1994.

20. L. V. Wang, "Mechanisms of ultrasonic modulation of multiply scattered coherent light: a monte carlo model," Opt. Lett. 26, pp. 1191-1193, 2001.

21. G. Yao and L. Wang, "Signal dependence and noise source in ultrasound-modulated optical tomography," Appl. Opt. 43, pp. 1320 - 1326, 2004.

22. U. Frisch, Wave propagation in random media, vol. 1 of Probabilistic methods in applied mathematics, pp. 75-198. Academic, New York, 1968.

23. Y. N. Barabanenkov, "Application of the smooth-perturbation method to the solution of general equations of multiple wave-scattering theory," Sov. Phys. JETP 27, pp. 954-959, 1968.

24. Y. N. Barabanenkov, A. G. Vinogradov, Yu. A. Kravtsov, and V. I. Tatarskii, "Application of the theory of multiple scattering of waves to the derivation of the radiation transfer equation for a statistically inhomogeneous medium," Radiophys. Quantum Electron. 15, pp. 1420-1425, 1972.

25. Yu. A. Kravtsov and L. A. Apresyan, Radiative transfer: new aspects of the old theory, vol. 36 of Progress in Optics, pp. 179-244. Elsevier, Amsterdam, 1996.

26. S. M. Rytov, Yu. A. Kravtsov, and V. I. Tatarskii, Principles of Statistical Radiophysics 3: Elements of Random Fields, Springer Verlag, Berlin Heidelberg, 1 ed., 1989.

27. S. M. Rytov, Yu. A. Kravtsov, and V. I. Tatarskii, Principles of Statistical Radiophysics 4: Wave Propagation Through Random Media, Springer Verlag, Berlin Heidelberg, 1 ed., 1989.

28. A. Ishimaru, Wave Propagation and Scattering in Random Media, Academic, New York, 1978.

29. L. Cohen, "Time-frequency distributions - a review," P. IEEE 77, pp. 941-981, 1989.

30. L. G. Henyey and J. L. Greenstein, "Diffuse radiation in the galaxy," Astrophys. J. 93, pp. 70-83, 1941.

31. L. V. Wang, S. L. Jacques, and L.-Q. Zheng, "Mcml - monte carlo modeling of photon transport in multilayered tissues," Comput. Methods Prog. Biomed. 47, pp. 131-146, 1995.

32. J. A. Jensen and N. B. Svendsen, "Calculation of pressure fields from arbitrarily shaped, apodized, and excited ultrasound transducers," IEEE Trans. Ultrason., Ferroelec., Freq. Contr. 39, pp. 262-267, 1992. 